\title{
ELEMENTARY SEPARATION BY ELEMENTARY EXPANSION
}

\author{
LEO MARCUS
}

\begin{abstract}
If $M, N$ are countable, nonisomorphic models, such that $N$ is not saturated and $M$ is prime, then there is an expansion $M^{*}$ of $M$, having the same elementary submodels as $M$, such that there is no expansion of $N$ elementarily equivalent to $M^{*}$.
\end{abstract}

The two terms in the title are newly coined here. The meaning of the first will be evident from Shelah's theorem quoted below and the second will be defined further on. Other model-theoretic notation and terminology is standard. The question considered in this note was motivated by the following unpublished result of Shelah (we include a sketch of the proof with the author's kind permission):

THEOREM 1 (Shelah). If $M, N$ are countable, nonisomorphic models, such that $N$ is not saturated, then there is a countable expansion $M^{*}$ of $M$ such that there is no expansion $N^{*}$ of $N$ with $N^{*} \equiv M^{*}$.

Sketch OF PROOF. Assume the type $p(x, \bar{a})=\left\{\varphi_{i}(x, \bar{a}): i<\omega\right\}$ is not realized in $N$, where $\bar{a}$ is a sequence of elements from $N, \varphi_{i+1} \rightarrow \varphi_{i}$. Let $M^{*}=\left(M, b_{i}^{M},<^{M}, F^{M}(x, \bar{z})\right)_{i<\omega}$ where the $b_{i}^{M}$ are names for all the elements of $M,<^{M}$ is an order relation such that $b_{i}^{M}<^{M} b_{i+1}^{M}$ for all $i<\omega$, and $F^{M}(x, \bar{z})$ is a function defined as follows:

If $M \vDash \exists x \varphi_{i}(x, \bar{c})$ then $M \vDash \varphi_{i}\left(F^{M}\left(b_{i}, \bar{c}\right), \bar{c}\right)$. If $M \sharp \exists x \varphi_{i}(x, \bar{c}), F^{M}\left(b_{i}, \bar{c}\right)$ is arbitrary.

Now assume $N^{*}=\left(N, b_{i}^{N},<^{N}, F^{N}(x, \bar{z})\right)_{i<\omega} \equiv M^{*}$. If $<^{N}$ is of order type $\omega$ then the map $b_{i}^{M} \mapsto b_{i}^{N}$ is an isomorphism between $M$ and $N$, contradiction. On the other hand if $b \in N$ is an element with $\omega<^{N}$ predecessors then $F^{N}(b, \bar{a})$ realizes $p$ in $N$, contradiction.

Notice that $M^{*}$ in the above proof is a minimal model, that is, $M_{1} \prec M^{*}$ $\Rightarrow M_{1}=M^{*}$. A natural question is: Can we separate $M$ from $N$ by an expansion which does not destroy all the proper elementary submodels of $M$, or better yet, does not destroy any?

Definition. $M^{*}$ is an elementary expansion of $M$ if for every $M_{1} \prec M, M_{1}$ is the universe of an elementary submodel of $M^{*}$.

Received by the editors December 27, 1975.

AMS (MOS) subject classifications (1970). Primary $02 \mathrm{H} 05$. 
THEOREM 2. If $M, N$ are countable, nonisomorphic models, such that $N$ is not saturated, and $M$ is prime, then there is an elementary expansion $M^{*}$ of $M$ such that there is no expansion $N^{*}$ of $N$ with $N^{*} \equiv M^{*}$.

We first prove two lemmas.

Definition. $M^{*}$ is a type-preserving expansion of $M$ if any two finite sequences which realize the same type in $M$ realize the same type in $M^{*}$.

LEMMA 1. If $M$ is prime, then any type-preserving expansion is an elementary expansion.

Proof. Easy.

Lemma 2. Let $M$ be prime, $p(\bar{x}), q(\bar{x}, y)$ nonatomic complete types such that $q(\bar{x}, y) \vdash p(\bar{x})$. Then there is an elementary expansion $M^{*}$ of $M$ such that if $N^{*} \equiv M^{*}$ then any sequence in $N^{*}$ realizing $p$ can be extended to a sequence in $N^{*}$ realizing $q$.

Proof. Let $p(\bar{x})=\left\{\varphi_{i}(x): i<\omega\right\}, q(\bar{x}, y)=\left\{\psi_{i}(\bar{x}, y): i<\omega\right\}$, where $\varphi_{i+1}$ $\rightarrow \varphi_{i}, \psi_{i+1} \rightarrow \psi_{i}$ : Consider the type $p^{*}(\bar{x})=\left\{\varphi_{i}^{*}(\bar{x}): i<\omega\right\}$ where $\varphi_{i}^{*}(\bar{x})$ $=\varphi_{i}(\bar{x}) \wedge \exists y \psi_{i}(\bar{x}, y)$. Clearly $p \equiv p^{*}$.

Now we define the relation $R(\bar{x}, y)$ on $M$. Let $\bar{a}$ be a sequence from $M$; there is $i<\omega$ such that $M \vDash \varphi_{i}^{*}(\bar{a}) \wedge \neg \varphi_{i+1}^{*}(\bar{a})$ since $p$ is omitted in $M$. Define $R^{M}(\bar{a}, b)$ if and only if $M \vDash \psi_{i}(\bar{a}, b)$. In other words, $R^{M}(\bar{x}, y) \leftrightarrow \forall i\left(\varphi_{i}^{*}(\bar{x})\right.$ $\left.\rightarrow \psi_{i}(\bar{x}, y)\right)$. Also it is clear that $(M, R) \vDash \forall \bar{x} \exists y R(\bar{x}, y)$.

Let $M^{*}=(M, R)$ and assume $N^{*} \equiv M^{*}, N^{*}$ realizes $p$. Let $\bar{c}$ realize $p$ in $N^{*}$, so $\bar{c}$ realizes $p^{*}$. Let $d$ be such that $N^{*} \vDash R(\bar{c}, d)$. Then $N^{*} \vDash \varphi_{i}^{*}(\bar{c})$ for all $i$, so $N^{*} \vDash \psi_{i}(\bar{c}, d)$ for all $i$, so $\bar{c}^{\wedge}\langle d\rangle$ realizes $q$.

We must now show that $M^{*}$ is an elementary expansion of $M$. By Lemma 1 , it is sufficient to show that $M^{*}$ is a type-preserving expansion of $M$. But this follows from the homogeneity of $M$ : If $\bar{a}$ and $\bar{b}$ are two sequences realizing the same type in $M$, then there is an automorphism of $M$ taking $\bar{a}$ onto $\bar{b}$. This automorphism also preserves $R$ since $R$ is definable by a sentence of $L_{\omega_{1}, \omega}$. Thus $\bar{a}$ and $\bar{b}$ realize the same type in $M^{*}$.

Proof of Theorem 2. Let $N$ be not saturated. Then there is a type $q_{1}(y)$ over a finite set $\bar{a}$ which is not realized in $N$. Since $N$ is not prime we may assume that $\bar{a}$ realizes a nonatomic type $p(\bar{x})$. Let $q(\bar{x}, y)=\left\{\psi_{i}(x, y): i<\omega\right\}$ where $\psi_{i}(\bar{a}, y) \in q_{1}(y)$. Then for this $p$ and $q$ the expansion $M^{*}$ given by Lemma 2 satisfies the requirements of the theorem.

Institute of Mathematics, The Hebrew University, Jerusalem, IsRael

Current address: Department of Mathematics, The University of California at Santa Barbara, Santa Barbara, California 93106 